\title{
The crystal structure of faustite and its copper analogue turquoise
}

\author{
U. KOLITSCH* AND G. Giester
}

Institut für Mineralogie und Kristallographie, Geozentrum, Universität Wien, Althanstr. 14, A-1090 Wien, Austria

\section{ABSTRACT}

The crystal structure of faustite, $\mathrm{ZnAl}_{6}\left(\mathrm{PO}_{4}\right)_{4}(\mathrm{OH})_{8} \cdot 4 \mathrm{H}_{2} \mathrm{O}$, was determined using single-crystal data (Mo- $K \alpha \mathrm{X}$-radiation, $\mathrm{CCD}$ area detector, 1624 unique reflections, $R 1=4.91 \%$, w $R 2=9.23 \%$ ), and compared with results of a reinvestigation of the structure of its copper analogue turquoise, $\mathrm{CuAl}_{6}\left(\mathrm{PO}_{4}\right)_{4}(\mathrm{OH})_{8} \cdot 4 \mathrm{H}_{2} \mathrm{O}(2737$ unique reflections, $R 1=2.81 \%$, w $R 2=6.90 \%)$. Both are isostructural and crystallize in space group $P \overline{\mathrm{l}}$, with $a=7.419(2)$ [turquoise: $7.410(1)$ ], $b=7.629(3)$ [7.633(1)], $c=$ 9.905(3) [9.904(1)] $\AA, \alpha=69.17(2)[68.42(1)], \beta=69.88(2)[69.65(1)], \gamma=64.98(2)[65.05(1)]^{\circ}, V=$ 462.2(3) $[460.27(10)] \AA^{3}$, and $Z=1$. The structure consists of distorted $M \mathrm{O}_{6}$ polyhedra $(M=\mathrm{Zn}, \mathrm{Cu})$, $\mathrm{AlO}_{6}$ octahedra and $\mathrm{PO}_{4}$ tetrahedra. By edge- and corner-sharing of these polyhedra a fairly dense three-dimensional framework is formed which is further strengthened by a system of hydrogen bonds. The metal atoms in the unique $M \mathrm{O}_{6}(M=\mathrm{Zn}$ or $\mathrm{Cu})$ polyhedron show a distorted $[2+2+2]$-coordination, the distortion being more pronounced in turquoise. About $10 \%$ of the $M$ site is vacant in both minerals. In turquoise, a previously undetected structural site with a very low occupancy of (possibly) $\mathrm{Cu}$ is present at the position $(1 / 2,0,1 / 2)$.

Kerwords: faustite, turquoise, crystal structure, crystal chemistry, phosphates.

\section{Introduction}

Faustite, $\mathrm{ZnAl}_{6}\left(\mathrm{PO}_{4}\right)_{4}(\mathrm{OH})_{8} \cdot 4 \mathrm{H}_{2} \mathrm{O}$ (Erd et al., $1953)$, is the rare $\mathrm{Zn}$ analogue of turquoise, $\mathrm{CuAl}_{6}\left(\mathrm{PO}_{4}\right)_{4}(\mathrm{OH})_{8} \cdot 4 \mathrm{H}_{2} \mathrm{O}$, with which it forms a solid solution series. Both belong to the turquoise group which also includes the members chalcosiderite, $\mathrm{CuFe}_{6}^{3+}\left(\mathrm{PO}_{4}\right)_{4}(\mathrm{OH})_{8} \cdot 4 \mathrm{H}_{2} \mathrm{O}$, aheylite, $\left(\mathrm{Fe}^{2-}, \mathrm{Zn}\right) \mathrm{Al}_{6}\left(\mathrm{PO}_{4}\right)_{4}(\mathrm{OH})_{8} \cdot 4 \mathrm{H}_{2} \mathrm{O}$ and planerite, $\square \mathrm{Al}_{6}\left(\mathrm{PO}_{4}\right)_{2}\left(\mathrm{PO}_{3} \mathrm{OH}\right)_{2}(\mathrm{OH})_{8} \cdot 4 \mathrm{H}_{2} \mathrm{O} \quad(\square=$ vacancy) (see recent review of Foord and Taggart, 1998 for further details). The crystal structure of faustite has not been verified or refined to date, although it was assumed to be isostructural with turquoise, which had been determined to be triclinic, with space group $P \overline{1}$ (Cid-Dresdner, 1965; $R=7 \%$ ).

Recently, the first occurrence of single, welldeveloped crystals of faustite has been reported

* E-mail: uwe.kolitsch@univie.ac.at from the Iron Monarch iron ore deposit in South Australia (Pring et al., 2000). The pale brownish rose to colourless, platy or prismatic faustite crystals (up to $0.1 \mathrm{~mm}$ ) are accompanied by hausmannite and kleemanite, $\mathrm{ZnAl}_{2}\left(\mathrm{PO}_{4}\right)_{2}(\mathrm{OH})_{2} \cdot 3 \mathrm{H}_{2} \mathrm{O}$. Chemical analyses have demonstrated (Pring et al., 2000) that the faustite contains little or no copper and only very small quantities of iron (probably $\mathrm{Fe}^{3+}$ substituting for $\mathrm{Al}$; see Foord and Taggart, 1998). The present article reports the refinement of the crystal structure of one of these faustite crystals. For comparison purposes, we also reinvestigated the crystal structure of turquoise because the earlier structure determination (Cid-Dresdner, 1965) reported unusually large ranges for the $\mathrm{Al}-\mathrm{O}$ and $\mathrm{P}-\mathrm{O}$ bond lengths.

\section{Experimental}

A very small chip cut from a colourless single crystal of faustite was selected for the structure analysis. The crystal chip with the approximate 


\section{U. KOLITSCH AND G. GIESTER}

dimensions $0.10 \times 0.05 \times 0.03 \mathrm{~mm}$ was mounted on a Nonius KappaCCD diffractometer equipped with a $300 \mu \mathrm{m}$ diameter capillary-optics collimator for improved resolution. Preliminary investigations gave a triclinic unit cell very similar to that reported for turquoise (CidDresdner, 1965). For the intensity data collection

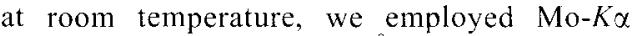
$X$-radiation $(\lambda=0.71073 \AA)$ and a detector distance of $28 \mathrm{~mm}$. Using $\varphi$ and $\omega$ as rotation axes (rotation width $2 \% 382$ data frames were aquired, each collected over $365 \mathrm{~s}$ (see Table 1 for further details). The data were processed using the Nonius program suite DENZO-SMN and corrected for Lorentz, polarization and background effects. Because of the small absorption coefficient, $\mu=2.12 \mathrm{~mm}^{-1}$, and the small size of the crystal chip used, no absorption correction was applied.
A pale bluish crystal of turquoise with the approximate dimensions $0.12 \times 0.08 \times 0.05 \mathrm{~mm}$ was chosen for the reinvestigation of the turquoise structure. The crystal was taken from a specimen showing well-developed, pseudorhombohedral crystals, also from Iron Monarch, South Australia. Semiquantitative energy-dispersive chemical analyses of three different turquoise crystals showed them to contain only traces of $Z_{n}$, $\mathrm{K}$ or $\mathrm{Ca}$ as impurity elements. The selected turquoise crystal was measured under very similar conditions (see above and Table 1). A triclinic unit cell was found which was very similar to that reported by Cid-Dresdner (1965). Again, no absorption correction was applied. Normalized structure factor statistics indicated the centrosymmetric space group $P \overline{1}$ for both faustite and turquoise crystals, in agreement with the observed crystal habits.

TABLE 1. Crystal data, data collection information, and refinement details for faustite and turquoise.

\begin{tabular}{|c|c|c|}
\hline & Faustite & Turquoise \\
\hline Formula (simplified) & $\mathrm{ZnAl}_{6}\left(\mathrm{PO}_{4}\right)_{4}(\mathrm{OH})_{8} \cdot 4 \mathrm{H}_{2} \mathrm{O}$ & $\mathrm{CuAl}_{6}\left(\mathrm{PO}_{4}\right)_{4}(\mathrm{OH})_{8} \cdot 4 \mathrm{H}_{2} \mathrm{O}$ \\
\hline Formula weight & 815.26 & 813.43 \\
\hline Space group & $P \overline{1}$ & $P \overline{1}$ \\
\hline$a, b, c(\AA)$ & $7.419(2), 7.629(3), 9.905(3)$ & $7.410(1), 7.633(1), 9.904(1)$ \\
\hline$\alpha, \beta, \gamma(0)$ & $69.17(2), 69.88(2), 64.98(2)$ & $68.42(1), 69.65(1), 65.05(1)$ \\
\hline$V\left(\mathrm{~A}^{3}\right), Z$ & $462.2(3) .1$ & $460.27(10), 1$ \\
\hline$F(000)$ & 408.0 & 407.0 \\
\hline$\mu\left(\mathrm{mm}^{-1}\right)$ & 2.12 & 1.98 \\
\hline$\rho_{\text {calc }}\left(\mathrm{g} \cdot \mathrm{cm}^{-3}\right)$ & 2.929 & 2.935 \\
\hline \multicolumn{3}{|l|}{ Data collection and refinement } \\
\hline Total no. of frames & 382 & 304 \\
\hline Collect. time per frame (s) & 365 & 220 \\
\hline Collection mode & sphere & sphere \\
\hline$\theta_{\max }\left({ }^{\circ}\right)$ & 30.0 (used: 25.03 ) & 30.49 \\
\hline$h, k, l$ ranges & $-8 \rightarrow 8,-9 \rightarrow 9,-11 \rightarrow 11$ & $-10 \rightarrow 10,-10 \rightarrow 10,-13 \rightarrow 13$ \\
\hline Total refls. measured & 4389 & 5193 \\
\hline Unique reflections & 1624 & 2737 \\
\hline$R 1(F), \mathrm{w} R 2\left(F^{2}\right)$ & $4.91 \%, 9.23 \% *$ & $2.81 \%, 6.90 \% \%^{\dagger}$ \\
\hline 'Observed' refls. & $1443\left[F_{\mathrm{o}}>4 \sigma\left(F_{\mathrm{o}}\right)\right]$ & $2437\left[F_{0}>4 \sigma\left(F_{0}\right)\right]$ \\
\hline Extinct. factor & - & $0.0173(16)$ \\
\hline No. of refined parameters & 211 & 213 \\
\hline 'Goodness of Fit' (GoF) & 1.212 & 1.106 \\
\hline$(\Delta / \sigma)_{\max }$ & 0.0001 & 0.0001 \\
\hline$\Delta \rho_{\min }, \Delta \rho_{\max }\left(\mathrm{e} / \AA^{3}\right)$ & $-0.49,0.52$ & $-0.60,0.75$ \\
\hline
\end{tabular}

Note: Unit-cell parameters were refined from all recorded reflections. Scattering factors for neutral atoms were employed in the refinement.

${ }^{*} \mathrm{w}=1 /\left[\sigma^{2}\left(F_{o}^{2}\right)+(0.02 \mathrm{P})^{2}+1.9 \mathrm{P}\right] ; \mathrm{P}=\left(\left[\max\right.\right.$ of $\left(0\right.$ or $\left.\left.\left.F_{o}^{2}\right)\right]+2 F_{c}^{2}\right) / 3$

${ }^{+} \mathrm{W}=1 /\left[\sigma^{2}\left(F_{o}^{2}\right)+(0.02 \mathrm{P})^{2}+0.5 \mathrm{P}\right] ; \mathbf{P}=\left(\left[\max\right.\right.$ of $\left(0\right.$ or $\left.\left.\left.F_{o}^{2}\right)\right]+2 F_{c}^{2}\right) / 3$ 


\section{CRYSTAL STRUCTURE OF FAUSTITE}

The positions of the metal and oxygen atoms in faustite were located using direct methods (SHELXS-97, Sheldrick, 1997a) and subsequent Fourier and difference Fourier syntheses. The deduced structure model is basically that of turquoise (Cid-Dresdner, 1965). Further difference Fourier syntheses revealed all $8 \mathrm{H}$ atoms present in the unit cell. The positions of the $\mathrm{H}$ atoms given here are those which are in fact bonded to the respective $\mathrm{O}$ atoms, and not the symmetry-equivalent positions as listed in CidDresdner (1965). Full-matrix least-squares refinement on $F^{2}$ (SHELXL-97, Sheldrick, 1997b), involving anisotropic displacement parameters for all non- $\mathrm{H}$ atoms, gave a discrepancy factor $R 1$ of $\sim 6 \%$. At this stage, residuals of the electron density were in the range of $\pm 1 \mathrm{e} / \AA^{3}$. The refinement of turquoise proceeded in the same way, with $R 1 \approx 3.5 \%$ in the later stages of the refinement and electron density residuals $<+1.2 \mathrm{e} / \AA^{3}$.

The approximate $\mathrm{Zn}: \mathrm{Cu}$ ratio in the faustite crystal chip measured was then determined after the data collection by semiquantitative energydispersive $X$-ray analysis, giving a value of $\sim 10: 1$. Additional point analyses of one small cluster of faustite crystals showed that the $\mathrm{Zn}: \mathrm{Cu}$ ratio can be fairly variable (probably even within a single crystal), ranging from $\sim 12: 1$ to $\sim 3: 1$. Very minor $\mathrm{Fe}$ was detected in both the measured crystal and the crystal cluster.

It is known that both turquoise and faustite form a complete solid solution series with planerite, $\square \mathrm{Al}_{6}\left(\mathrm{PO}_{4}\right)_{2}\left(\mathrm{PO}_{3} \mathrm{OH}\right)_{2}(\mathrm{OH})_{8} \cdot 4 \mathrm{H}_{2} \mathrm{O}$ (i.e. a member of the turquoise group with dominant vacancies on the transition metal site), and that cuprian planerite is in fact more common than turquoise (Foord and Taggart, 1988). Accordingly, we refined the occupancy of the $M$ $(M=\mathrm{Zn}, \mathrm{Cu})$ site in faustite and turquoise, neglecting the very minor $\mathrm{Cu}-\mathrm{for}-\mathrm{Zn}$ substitution in faustite. The occupancy factors obtained were $0.927(5)$ and $0.874(2)$ for faustite and turquoise, respectively, indicating that the $M$ site contains 7 and $13 \%$ vacancies. If the $M$ site was constrained to be fully occupied, the $R$ factors increased considerably. During the last refinement stage for turquoise, the difference Fourier map showed a distinct peak at the position $(1 / 2,0,1 / 2)$. Considering the site coordination and bond lengths, a minor amount of $\mathrm{Cu}$ was preliminarily assigned to this position and $U_{\text {eq }}$ was fixed at 0.02 . Refinement of the occupancy factor gave a very low value, $0.0193(12)$. This site is discussed further below.
The final refinement cycle for faustite led to $R 1=$ $4.91 \%, \quad w R 2=9.23 \%$. Final $R$ factors for turquoise were $R 1=2.81 \%, \mathrm{w} R 2=6.90 \%$. Residual electron densities in both crystals were $< \pm 1 \mathrm{e} / \AA^{3}$. The final positional and displacement parameters are presented in Tables 2 and 3, and selected bond lengths and angles in Table 4. Probable hydrogen bonds are listed in Table 5. Results of empirical bond-valence calculations (Brown and Altermatt, 1985; Brese and O'Keeffe, 1991; Brown, 1996) are given in Table 6. Lists of observed and calculated structure factors (Table 7) have been deposited with the Editor.

\section{Discussion}

\section{Structure connectivity and cation coordinations}

The structure contains distorted $M \mathrm{O}_{6}$ polyhedra $(M=\mathrm{Zn}, \mathrm{Cu}), \mathrm{AlO}_{6}$ octahedra and $\mathrm{PO}_{4}$ tetrahedra. The dominant structure element is an $\left[\mathrm{Al}_{2} \mathrm{MAl}_{2}\left(\mathrm{O}, \mathrm{OH}, \mathrm{H}_{2} \mathrm{O}\right)_{18}\right]$ polyhedral cluster formed by a central $M \mathrm{O}_{6}$ polyhedron sharing four edges with two edge-sharing $\mathrm{Al}(1) \mathrm{O}_{6}$ $\mathrm{Al}(2) \mathrm{O}_{6}$ dimers (Fig. 1). The $\mathrm{AlO}_{6}$ octahedra in the cluster are corner-linked to the $\mathrm{Al}(3) \mathrm{O}_{6}$ octahedron which shares $\mathrm{Oh}(9)$ with $\mathrm{Al}(1) \mathrm{O}_{6}$ and $M \mathrm{O}_{6}$, and $\mathrm{Oh}(12)$ with $\mathrm{Al}(2) \mathrm{O}_{6}$ and $M \mathrm{O}_{6}$. Further corner-linkage via the two non-equivalent

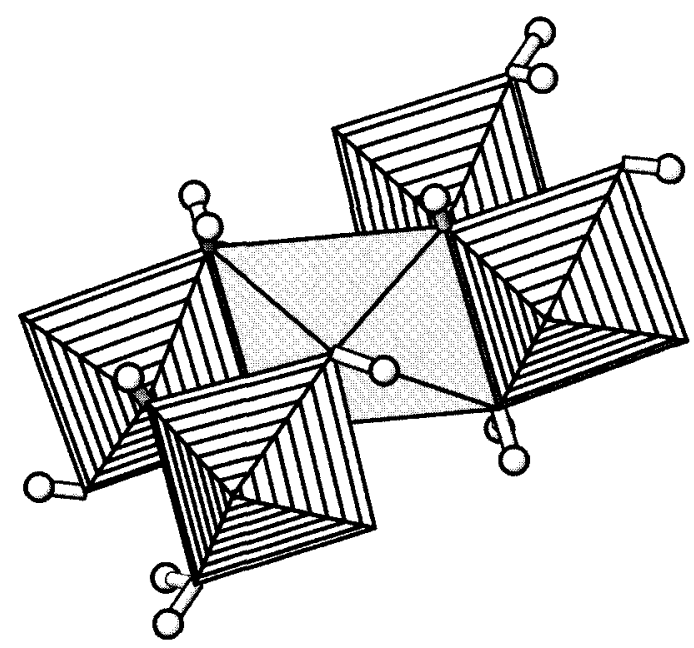

Fig. 1. The dominant element in the faustite (and turquoise) structure: an $\left[\mathrm{Al}_{2} \mathrm{MAl}_{2}\left(\mathrm{O}, \mathrm{OH}, \mathrm{H}_{2} \mathrm{O}\right)_{18}\right]$ polyhedral cluster formed by a central (shaded) $M \mathrm{O}_{6}$ polyhedron $(M=\mathrm{Zn}, \mathrm{Cu})$ which shares four of its edges with two edge-sharing $\mathrm{Al}(1) \mathrm{O}_{6}-\mathrm{Al}(2) \mathrm{O}_{6}$ dimers. All drawings were done using ATOMS (Shape Software, 1999). 
TABLE 2. Fractional atomic coordinates and isotropic displacement parameters for faustite and turquoise (every second row).

\begin{tabular}{|c|c|c|c|c|c|}
\hline Atom & $x$ & $y$ & $z$ & $U_{\mathrm{eq}} / U$ & Occupancy \\
\hline $\mathrm{Zn}$ & 0 & 0 & 0 & $0.0301(4)$ & $0.927(5)$ \\
\hline $\mathrm{Cu}$ & 0 & 0 & 0 & $0.00875(13)$ & $0.874(2)$ \\
\hline \multirow{2}{*}{ Al1 } & $0.2835(2)$ & $0.1804(2)$ & $0.74960(16)$ & $0.0127(4)$ & \\
\hline & $0.28321(9)$ & $0.17672(8)$ & $0.75262(6)$ & $0.00739(14)$ & \\
\hline \multirow[t]{2}{*}{$\mathrm{Al} 2$} & $0.7478(2)$ & $0.1861(2)$ & $0.27319(16)$ & $0.0123(4)$ & \\
\hline & $0.75315(9)$ & $0.18560(8)$ & $0.27287(6)$ & $0.00683(14)$ & \\
\hline \multirow[t]{2}{*}{$\mathrm{Al} 3$} & $0.2431(2)$ & $0.5022(2)$ & $0.24472(16)$ & $0.0083(3)$ & \\
\hline & $0.24489(8)$ & $0.50221(7)$ & $0.24459(6)$ & $0.00571(14)$ & \\
\hline \multirow[t]{2}{*}{$\mathrm{P}(1)$} & $0.35248(19)$ & $0.38778(19)$ & $0.94179(14)$ & $0.0121(3)$ & \\
\hline & $0.35046(7)$ & $0.38634(7)$ & $0.94324(5)$ & $0.00608(12)$ & \\
\hline \multirow[t]{2}{*}{$\mathrm{P}(2)$} & $0.84463(19)$ & $0.38499(19)$ & $0.45620(14)$ & $0.0119(3)$ & \\
\hline & $0.84272(7)$ & $0.38707(7)$ & $0.45631(5)$ & $0.00540(12)$ & \\
\hline \multirow[t]{2}{*}{$O(1)$} & $0.0697(5)$ & $0.3606(5)$ & $0.3841(4)$ & $0.0147(8)$ & \\
\hline & $0.0680(2)$ & $0.3629(2)$ & $0.38468(15)$ & $0.0089(3)$ & \\
\hline \multirow[t]{2}{*}{$O(2)$} & $0.8067(5)$ & $0.3433(5)$ & $0.6245(4)$ & $0.0142(8)$ & \\
\hline & $0.8045(2)$ & $0.34485(19)$ & $0.62581(14)$ & $0.0078(3)$ & \\
\hline \multirow{2}{*}{$O(3)$} & $0.2799(5)$ & $0.3545(5)$ & $0.1113(4)$ & $0.0151(8)$ & \\
\hline & $0.2767(2)$ & $0.3558(2)$ & $0.11311(14)$ & $0.0084(3)$ & \\
\hline \multirow[t]{2}{*}{ Ow(4) } & $0.0667(6)$ & $0.0611(6)$ & $0.1897(4)$ & $0.0208(9)$ & \\
\hline & $0.0632(2)$ & $0.0661(3)$ & $0.19825(18)$ & $0.0130(3)$ & \\
\hline \multirow[t]{2}{*}{$\mathrm{Oh}(5)$} & $0.2379(6)$ & $0.0722(6)$ & $0.6281(4)$ & $0.0145(8)$ & \\
\hline & $0.2377(2)$ & $0.0732(2)$ & $0.62792(17)$ & $0.0081(3)$ & \\
\hline \multirow[t]{2}{*}{$\mathrm{Oh}(6)$} & $0.7325(6)$ & $0.0794(6)$ & $0.1266(5)$ & $0.0140(9)$ & \\
\hline & $0.7355(2)$ & $0.0828(2)$ & $0.12361(16)$ & $0.0075(3)$ & \\
\hline \multirow[t]{2}{*}{$O(7)$} & $0.2957(5)$ & $0.4056(5)$ & $0.6052(4)$ & $0.0148(8)$ & \\
\hline & $0.2962(2)$ & $0.40354(19)$ & $0.60706(14)$ & $0.0089(3)$ & \\
\hline \multirow[t]{2}{*}{$O(8)$} & $0.3261(5)$ & $0.2269(5)$ & $0.9022(4)$ & $0.0149(8)$ & \\
\hline & $0.3253(2)$ & $0.2230(2)$ & $0.90554(15)$ & $0.0101(3)$ & \\
\hline \multirow[t]{2}{*}{$\mathrm{Oh}(9)$} & $0.9882(5)$ & $0.2880(5)$ & $0.8390(4)$ & $0.0146(8)$ & \\
\hline & $0.9863(2)$ & $0.2807(2)$ & $0.84408(17)$ & $0.0088(3)$ & \\
\hline \multirow[t]{2}{*}{$\mathrm{Ow}(10)$} & $0.5758(5)$ & $0.0513(6)$ & $0.6830(4)$ & $0.0167(8)$ & \\
\hline & $0.5741(2)$ & $0.0494(2)$ & $0.68631(17)$ & $0.0113(3)$ & \\
\hline \multirow[t]{2}{*}{$\mathrm{O}(11)$} & $0.7823(5)$ & $0.4059(5)$ & $0.1313(4)$ & $0.0152(8)$ & \\
\hline & $0.7848(2)$ & $0.4090(2)$ & $0.13125(15)$ & $0.0093(3)$ & \\
\hline \multirow[t]{2}{*}{$\operatorname{Oh}(12)$} & $0.4632(6)$ & $0.2949(5)$ & $0.3269(4)$ & $0.0146(8)$ & \\
\hline & $0.4649(2)$ & $0.2940(2)$ & $0.32633(17)$ & $0.0080(3)$ & \\
\hline $\mathrm{O}(13)$ & $0.7924(5)$ & $0.2274(5)$ & $0.4283(3)$ & $0.0128(8)$ & \\
\hline & $0.7883(2)$ & $0.22916(19)$ & $0.43134(14)$ & $0.0080(3)$ & \\
\hline$O(14)$ & $0.5786(5)$ & $0.3666(5)$ & $0.8964(4)$ & $0.0145(8)$ & \\
\hline & $0.5771(2)$ & $0.3662(2)$ & $0.89794(14)$ & $0.0082(3)$ & \\
\hline $\mathrm{H}(1)$ & $0.133(13)$ & $-0.055(14)$ & $0.238(10)$ & $0.08(3)$ & \\
\hline & $0.128(5)$ & $-0.028(5)$ & $0.224(4)$ & $0.032(9)$ & \\
\hline $\mathrm{H}(2)$ & $0.148(17)$ & $0.111(18)$ & $0.184(13)$ & $0.13(5)$ & \\
\hline & $0.116(5)$ & $0.132(6)$ & $0.176(4)$ & $0.040(10)$ & \\
\hline $\mathrm{H}(3)$ & $0.653(11)$ & $0.111(11)$ & $0.608(8)$ & $0.05(2)$ & \\
\hline & $0.628(5)$ & $0.117(5)$ & $0.609(4)$ & $0.040(9)$ & \\
\hline $\mathrm{H}(4)$ & $0.607(12)$ & $-0.067(12)$ & $0.689(9)$ & $0.06(3)$ & \\
\hline & $0.608(5)$ & $-0.057(5)$ & $0.679(3)$ & $0.035(9)$ & \\
\hline $\mathrm{H}(5)$ & $0.185(10)$ & $0.128(9)$ & $0.580(7)$ & $0.02(2)$ & \\
\hline & $0.204(5)$ & $0.129(5)$ & $0.573(3)$ & $0.023(9)$ & \\
\hline $\mathrm{H}(6)$ & $0.671(9)$ & $0.135(9)$ & $0.091(7)$ & $0.01(2)$ & \\
\hline & $0.691(4)$ & $0.143(4)$ & $0.074(3)$ & $0.018(8)$ & \\
\hline$H(7)$ & $0.984(8)$ & $0.339(8)$ & $0.895(6)$ & $0.017(16)$ & \\
\hline & $0.987(5)$ & $0.318(5)$ & $0.889(4)$ & $0.029(10)$ & \\
\hline $\mathrm{H}(8)$ & $0.444(9)$ & $0.315(9)$ & $0.390(7)$ & $0.02(2)$ & \\
\hline & $0.448(4)$ & $0.287(4)$ & $0.397(3)$ & $0.021(8)$ & \\
\hline$X$ (turqu.)* & 0.5 & 0 & 0.5 & 0.02 & $\sim 0.03$ \\
\hline
\end{tabular}

Note: Oxygens of hydroxyl groups are designated as Oh, those of water molecules as Ow.

* $U$ was fixed (for possible identity of $X$ see text). Given occupancy refers to $X=\mathrm{Cu}$ 
CRYSTAL STRUCTURE OF FAUSTITE

TABlE 3. Anisotropic displacement parameters for faustite and turquoise (every second row).

\begin{tabular}{|c|c|c|c|c|c|c|}
\hline Atom & $U_{11}$ & $U_{22}$ & $U_{33}$ & $U_{23}$ & $U_{13}$ & $U_{12}$ \\
\hline $\mathrm{Zn}$ & $0.0271(7)$ & $0.0217(6)$ & $0.0263(7)$ & $-0.0087(5)$ & $0.0128(5)$ & $-0.0069(5)$ \\
\hline $\mathrm{Cu}$ & $0.0074(2)$ & $0.00798(19)$ & $0.0066(2)$ & $-0.00188(14)$ & $0.00203(13)$ & $-0.00159(13)$ \\
\hline \multirow[t]{2}{*}{ All } & $0.0145(8)$ & $0.0107(7)$ & $0.0134(8)$ & $-0.0031(6)$ & $-0.0038(6)$ & $-0.0043(6)$ \\
\hline & $0.0102(3)$ & $0.0059(3)$ & $0.0073(3)$ & $-0.0011(2)$ & $-0.0030(2)$ & $-0.0036(2)$ \\
\hline \multirow[t]{2}{*}{$\mathrm{Al} 2$} & $0.0151(8)$ & $0.0108(7)$ & $0.0116(8)$ & $-0.0024(6)$ & $-0.0039(6)$ & $-0.0044(6)$ \\
\hline & $0.0081(3)$ & $0.0061(3)$ & $0.0071(3)$ & $-0.0016(2)$ & $-0.0024(2)$ & $-0.0029(2)$ \\
\hline \multirow[t]{2}{*}{$\mathrm{A} 13$} & $0.0079(7)$ & $0.0072(7)$ & $0.0097(7)$ & $-0.0031(6)$ & $-0.0020(6)$ & $-0.0016(6)$ \\
\hline & $0.0061(3)$ & $0.0059(3)$ & $0.0053(3)$ & $-0.0020(2)$ & $-0.0006(2)$ & $-0.0023(2)$ \\
\hline \multirow[t]{2}{*}{$P(1)$} & $0.0130(7)$ & $0.0117(7)$ & $0.0125(7)$ & $-0.0040(5)$ & $-0.0032(5)$ & $-0.0040(5)$ \\
\hline & $0.0066(2)$ & $0.0066(2)$ & $0.0058(2)$ & $-0.00183(16)$ & $-0.00136(16)$ & $-0.00288(17)$ \\
\hline \multirow[t]{2}{*}{$\mathrm{P}(2)$} & $0.0127(7)$ & $0.0112(7)$ & $0.0124(7)$ & $-0.0034(5)$ & $-0.0037(5)$ & $-0.0037(5)$ \\
\hline & $0.0059(2)$ & $0.0057(2)$ & $0.0053(2)$ & $-0.00167(16)$ & $-0.00111(16)$ & $-0.00262(17)$ \\
\hline \multirow[t]{2}{*}{$O(1)$} & $0.0143(18)$ & $0.0126(17)$ & $0.0153(18)$ & $-0.0024(15)$ & $-0.0032(15)$ & $-0.0039(15)$ \\
\hline & $0.0071(6)$ & $0.0103(6)$ & $0.0086(6)$ & $-0.0022(5)$ & $-0.0002(5)$ & $-0.0037(5)$ \\
\hline \multirow[t]{2}{*}{$O(2)$} & $0.0179(19)$ & $0.0132(18)$ & $0.0127(18)$ & $-0.0036(15)$ & $-0.0035(15)$ & $-0.0061(15)$ \\
\hline & $0.0094(6)$ & $0.0084(6)$ & $0.0057(6)$ & $-0.0033(5)$ & $-0.0008(5)$ & $-0.0029(5)$ \\
\hline \multirow[t]{2}{*}{$O(3)$} & $0.0182(18)$ & $0.0146(18)$ & $0.0138(18)$ & $-0.0050(15)$ & $-0.0008(15)$ & $-0.0079(15)$ \\
\hline & $0.0113(7)$ & $0.0095(6)$ & $0.0050(6)$ & $-0.0030(5)$ & $-0.0002(5)$ & $-0.0047(5)$ \\
\hline \multirow[t]{2}{*}{ Ow(4) } & $0.020(2)$ & $0.020(2)$ & $0.022(2)$ & $-0.0044(18)$ & $-0.0066(17)$ & $-0.0053(19)$ \\
\hline & $0.0130(7)$ & $0.0120(8)$ & $0.0146(8)$ & $0.0003(6)$ & $-0.0057(6)$ & $-0.0060(7)$ \\
\hline \multirow[t]{2}{*}{$\operatorname{Oh}(5)$} & $0.018(2)$ & $0.0122(19)$ & $0.014(2)$ & $-0.0030(17)$ & $-0.0082(17)$ & $-0.0031(16)$ \\
\hline & $0.0119(7)$ & $0.0068(6)$ & $0.0069(7)$ & $0.0000(5)$ & $-0.0054(5)$ & $-0.0033(5)$ \\
\hline \multirow[t]{2}{*}{$\operatorname{Oh}(6)$} & $0.015(2)$ & $0.013(2)$ & $0.014(2)$ & $-0.0023(17)$ & $-0.0056(18)$ & $-0.0032(17)$ \\
\hline & $0.0087(6)$ & $0.0069(6)$ & $0.0069(7)$ & $-0.0005(5)$ & $-0.0041(5)$ & $-0.0020(5)$ \\
\hline \multirow[t]{2}{*}{$O(7)$} & $0.0161(19)$ & $0.0100(17)$ & $0.0182(19)$ & $-0.0037(15)$ & $-0.0041(15)$ & $-0.0042(15)$ \\
\hline & $0.0094(7)$ & $0.0064(6)$ & $0.0089(6)$ & $-0.0009(5)$ & $-0.0022(5)$ & $-0.0019(5)$ \\
\hline \multirow[t]{2}{*}{$O(8)$} & $0.0193(19)$ & $0.0131(18)$ & $0.0159(18)$ & $-0.0021(15)$ & $-0.0076(15)$ & $-0.0071(15)$ \\
\hline & $0.0142(7)$ & $0.0083(6)$ & $0.0109(7)$ & $-0.0027(5)$ & $-0.0054(5)$ & $-0.0044(5)$ \\
\hline \multirow[t]{2}{*}{$\mathrm{Oh}(9)$} & $0.017(2)$ & $0.0152(19)$ & $0.014(2)$ & $-0.0084(16)$ & $-0.0025(15)$ & $-0.0047(15)$ \\
\hline & $0.0085(7)$ & $0.0104(7)$ & $0.0092(7)$ & $-0.0059(5)$ & $-0.0001(5)$ & $-0.0035(5)$ \\
\hline \multirow[t]{2}{*}{ Ow(10) } & $0.018(2)$ & $0.013(2)$ & $0.018(2)$ & $-0.0016(17)$ & $-0.0023(16)$ & $-0.0074(17)$ \\
\hline & $0.0096(7)$ & $0.0092(7)$ & $0.0129(7)$ & $-0.0017(5)$ & $-0.0006(5)$ & $-0.0039(6)$ \\
\hline \multirow[t]{2}{*}{$O(11)$} & $0.0143(18)$ & $0.0123(18)$ & $0.0171(19)$ & $-0.0016(15)$ & $-0.0051(15)$ & $-0.0033(15)$ \\
\hline & $0.0091(6)$ & $0.0075(6)$ & $0.0099(7)$ & $-0.0008(5)$ & $-0.0024(5)$ & $-0.0024(5)$ \\
\hline \multirow[t]{2}{*}{$\mathrm{Oh}(12)$} & $0.015(2)$ & $0.015(2)$ & $0.013(2)$ & $-0.0062(17)$ & $-0.0024(16)$ & $-0.0034(15)$ \\
\hline & $0.0078(6)$ & $0.0092(6)$ & $0.0056(7)$ & $-0.0025(5)$ & $-0.0014(5)$ & $-0.0013(5)$ \\
\hline \multirow[t]{2}{*}{$O(13)$} & $0.0178(18)$ & $0.0124(17)$ & $0.0095(18)$ & $-0.0021(14)$ & $-0.0032(14)$ & $-0.0067(15)$ \\
\hline & $0.0123(7)$ & $0.0078(6)$ & $0.0079(6)$ & $-0.0025(5)$ & $-0.0038(5)$ & $-0.0051(5)$ \\
\hline \multirow[t]{2}{*}{$O(14)$} & $0.0112(18)$ & $0.0149(18)$ & $0.0174(19)$ & $-0.0039(15)$ & $-0.0036(14)$ & $-0.0043(14)$ \\
\hline & $0.0065(6)$ & $0.0091(6)$ & $0.0078(6)$ & $-0.0005(5)$ & $-0.0016(5)$ & $-0.0030(5)$ \\
\hline
\end{tabular}

$\mathrm{PO}_{4}$ tetrahedra forms a three-dimensional framework. Hydrogen bonds provide additional links between the polyhedral components (see also below). For further details and alternative descriptions of the connectivity, the reader is referred to Cid-Dresdner (1965) and the description of the structure of chalcosiderite, $\mathrm{CuFe}_{6}^{3+}\left(\mathrm{PO}_{4}\right)_{4}(\mathrm{OH})_{8} \cdot 4 \mathrm{H}_{2} \mathrm{O}$, the $\mathrm{Fe}$ analogue of turquoise (Giuseppetti et al., 1989).
In both faustite and turquoise the transition metal atoms show a $[2+2+2]$-coordination, which is, however, less pronounced in faustite $(2 \times 1.921$, $2 \times 2.197$ and $2 \times 2.335 \AA$ ) than in turquoise ( $2 \mathrm{x}$ $1.895,2 \times 2.121$ and $2 \times 2.427 \AA$ ). In chalcosiderite, $\mathrm{CuFe}_{6}^{3+}\left(\mathrm{PO}_{4}\right)_{4}(\mathrm{OH})_{8} \cdot 4 \mathrm{H}_{2} \mathrm{O}$, the $\mathrm{Cu}$ atom shows a comparable $[2+2+2]$-coordination $(2 \mathrm{x} 1.914,2 \mathrm{x}$ 2.079 and $2 \times 2.484 \AA$; Giuseppetti et al., 1989). The bond distances in turquoise are in accordance 


\section{U. KOLITSCH AND G. GIESTER}

TABLE 4. Selected bond distances $(\AA)$ for the coordination polyhedra in faustite and turquoise $(M=\mathrm{Zn}$ and $\mathrm{Cu}$, respectively).

\begin{tabular}{|c|c|c|c|c|c|}
\hline & Faustite & Turquoise & & Faustite & Turquoise \\
\hline$M-\mathrm{Oh}(6) \times 2$ & $1.921(4)$ & $1.8947(14)$ & $\mathrm{Al}(1)-\mathrm{O}(7)$ & $1.817(4)$ & $1.8192(14)$ \\
\hline- Oh $(9) \times 2$ & $2.196(4)$ & $2.1209(15)$ & $-\mathrm{O}(8)$ & $1.821(4)$ & $1.8240(14)$ \\
\hline$-O w(4) \times 2$ & $2.334(4)$ & $2.4272(16)$ & $-\mathrm{Oh}(5)$ & $1.862(4)$ & $1.8682(15)$ \\
\hline \multirow[t]{4}{*}{$<M-\mathrm{O}>$} & 2.15 & 2.148 & $-\mathrm{Ow}(10)$ & $1.944(4)$ & $1.9323(16)$ \\
\hline & & & $-\mathrm{Oh}(6)$ & $1.953(4)$ & $1.9467(16)$ \\
\hline & & & $-\mathrm{Oh}(9)$ & $1.980(4)$ & $1.9887(16)$ \\
\hline & & & $<\mathrm{Al}(1)-\mathrm{O}>$ & 1.896 & 1.897 \\
\hline$P(1)-O(8)$ & $1.516(3)$ & $.5209(14)$ & & & \\
\hline$-O(11)$ & $1.536(4)$ & $1.5305(14)$ & $\mathrm{Al}(2)-\mathrm{O}(11)$ & $1.814(4)$ & $1.8152(14)$ \\
\hline$-O(14)$ & $1.532(4)$ & $1.5341(14)$ & $-O(13)$ & $1.831(3)$ & $1.8383(14)$ \\
\hline$-O(3)$ & $1.538(4)$ & $1.5378(13)$ & $-\mathrm{Oh}(5)$ & $1.837(4)$ & $1.8412(16)$ \\
\hline \multirow{2}{*}{$\langle\mathrm{P}(1)-\mathrm{O}\rangle$} & 1.531 & 1.531 & $-\mathrm{Oh}(12)$ & $1.868(4)$ & $1.8888(15)$ \\
\hline & & & $-\mathrm{Oh}(6)$ & $1.954(4)$ & $1.9710(15)$ \\
\hline$P(2)-O(1)$ & $1.531(4)$ & $1.5280(14)$ & $-O w(4)$ & $2.121(4)$ & $2.0492(17)$ \\
\hline$-O(7)$ & $1.531(3)$ & $1.5291(14)$ & $\langle\mathrm{Al}(2)-\mathrm{O}\rangle$ & 1.904 & 1.901 \\
\hline$-O(2)$ & $1.528(3)$ & $1.5343(14)$ & & & \\
\hline$-O(13)$ & $1.541(3)$ & $1.5407(13)$ & $\mathrm{Al}(3)-\mathrm{O}(14)$ & $1.887(4)$ & $1.8788(14)$ \\
\hline \multirow[t]{6}{*}{$\langle\mathrm{P}(2)-\mathrm{O}\rangle$} & 1.533 & 1.533 & $-\mathrm{O}(2)$ & $1.895(3)$ & $1.8841(14)$ \\
\hline & & & $-O(1)$ & $1.903(4)$ & $1.8999(14)$ \\
\hline & & & $-O(3)$ & $1.908(3)$ & $1.9040(14)$ \\
\hline & & & $-\mathrm{Oh}(12)$ & $1.909(4)$ & $1.9063(15)$ \\
\hline & & & $-\mathrm{Oh}(9)$ & $1.977(4)$ & $2.0108(16)$ \\
\hline & & & $\langle\mathrm{Al}(3)-\mathrm{O}\rangle$ & 1.913 & 1.914 \\
\hline
\end{tabular}

TABLE 5. Probable hydrogen bonds $(\AA)$ in faustite and turquoise.

\begin{tabular}{ccc}
\hline & Faustite & Turquoise \\
\hline $\mathrm{Ow}(4)-\mathrm{H}(1) \cdots \mathrm{O}(2)$ & $2.913(6)$ & $2.906(2)$ \\
$-\mathrm{H}(1) \cdots \mathrm{O}(10)$ & $2.995(6)$ & $2.952(2)$ \\
$\mathrm{Ow}(4)-\mathrm{H}(2) \cdots \mathrm{O}(3)$ & $3.005(6)$ & $2.961(2)$ \\
$-\mathrm{H}(2) \cdots \mathrm{O}(8)$ & $3.023(5)$ & $3.020(2)$ \\
$\mathrm{Ow}(10)-\mathrm{H}(3) \cdots \mathrm{O}(13)$ & $2.716(5)$ & $2.702(2)$ \\
$\mathrm{Ow}(10)-\mathrm{H}(4) \cdots \mathrm{O}(12)$ & $2.814(6)$ & $2.806(2)$ \\
$-\mathrm{H}(4) \cdots \mathrm{O}(3)$ & $3.015(5)$ & $2.983(2)$ \\
$\mathrm{Oh}(5)-\mathrm{H}(5) \cdots \mathrm{O}(1)$ & $2.865(6)$ & $2.855(2)$ \\
$-\mathrm{H}(5) \cdots \mathrm{O}(2)$ & $2.990(5)$ & $3.001(2)$ \\
$\mathrm{Oh}(6)-\mathrm{H}(6) \cdots \mathrm{O}(14)$ & $2.724(6)$ & $2.685(2)$ \\
$\mathrm{Oh}(9)-\mathrm{H}(7) \cdots \mathrm{O}(3)$ & $2.707(5)$ & $2.720(2)$ \\
$-\mathrm{H}(7) \cdots \mathrm{O}(11)$ & $3.029(5)$ & $3.020(2)$ \\
$\mathrm{Oh}(12)-\mathrm{H}(8) \cdots \mathrm{O}(7)$ & $2.875(5)$ & $2.896(2)$ \\
& & \\
\hline
\end{tabular}

Note: Values given are $\mathrm{O}-\mathrm{O}$ distances. Refined (unrestrained) donor-hydrogen distances range between 0.59 and $0.88 \AA$ (faustite), and 0.69 and $0.83 \AA$ (turquoise) with the bimodal distribution of $\mathrm{Cu}-\mathrm{O}$ distances (maxima at 1.97 and $2.44 \AA$ ) observed for JahnTeller-distorted $\mathrm{Cu}^{2+}-\mathrm{O}$ polyhedra in copper oxysalt minerals (Eby and Hawthorne, 1993). The atomic environment of $\mathrm{Cu}$ in turquoise is in accordance with published electron paramagnetic resonance and optical absorption spectra (Sharma et al., 1988). Bond-valence sums for $\mathrm{Zn}$ and $\mathrm{Cu}$ are very close to ideal values (Table 6).

The partially occupied site in turquoise at $(1 / 2,0,1 / 2)$, on which a very minor amount of $\mathrm{Cu}$ was assumed to be located, has bond distances to nearest oxygens compatible with a $[2+2+2]-$ coordination of $\mathrm{Cu}, 2 \times 1.89,2 \times 2.25,2 \times 2.29 \AA$. The environment of this site is also very similar to that of the $M$ site (Fig. 2). However, trace impurity cations such as $\mathrm{Na}$ or $\mathrm{Ca}$, or water molecules could occupy the site, if one considers the possibility of positional disorder of these cations. No residual electron density was observed for faustite. Occupancy of the additional site in turquoise may be correlated with the lower partial occupancy of the principal $\mathrm{Cu}$ site at $(0,0,0)$. According to Foord and Taggart (1988), 


\section{CRYSTAL STRUCTURE OF FAUSTITE}

TABLE 6. Results of empirical bond-valence calculations for faustite and turquoise; for the latter only sum values (in brackets) are given.

\begin{tabular}{|c|c|c|c|c|c|c|c|}
\hline & $M$ & $\operatorname{Al}(1)$ & $\mathrm{Al}(2)$ & $\mathrm{Al}(3)$ & $P(1)$ & $\mathrm{P}(2)$ & Sum \\
\hline$O(1)$ & - & - & - & 0.506 & - & 1.218 & $1.72(1.74)$ \\
\hline$O(2)$ & - & - & - & 0.517 & - & 1.228 & $1.75(1.74)$ \\
\hline$O(3)$ & - & - & - & 0.499 & 1.195 & - & $1.69(1.70)$ \\
\hline Ow(4) & $\begin{array}{l}0.182 \\
\downarrow \times 2\end{array}$ & - & 0.281 & - & - & - & $0.65(0.47)$ \\
\hline $\mathrm{Oh}(5)$ & - & 0.565 & 0.605 & - & - & - & $1.17(1.15)$ \\
\hline $\operatorname{Oh}(6)$ & $\begin{array}{c}0.556 \\
\downarrow \times 2\end{array}$ & 0.442 & 0.441 & - & - & - & $1.44(1.43)$ \\
\hline $\mathrm{O}(7)$ & - & 0.638 & - & - & - & 1.218 & $1.86(1.86)$ \\
\hline$O(8)$ & - & 0.632 & - & - & 1.269 & - & $1.90(1.88)$ \\
\hline $\operatorname{Oh}(9)$ & $\begin{array}{c}0.265 \\
\downarrow \times 2\end{array}$ & 0.411 & - & 0.414 & - & - & $1.09(1.08)$ \\
\hline Ow(10) & - & 0.453 & - & - & - & - & $0.45(0.47)$ \\
\hline$O(11)$ & - & - & 0.644 & - & 1.202 & - & $1.85(1.69)$ \\
\hline $\operatorname{Oh}(12)$ & - & - & 0.556 & 0.498 & - & - & $1.05(1.03)$ \\
\hline$O(13)$ & - & - & 0.615 & - & - & 1.186 & $1.80(1.79)$ \\
\hline$O(14)$ & - & - & - & 0.528 & 1.215 & - & $1.74(1.75)$ \\
\hline Sum & $\begin{array}{c}2.01 \\
(1.99)\end{array}$ & $\begin{array}{c}3.14 \\
(3.14)\end{array}$ & $\begin{array}{c}3.14 \\
(3.13)\end{array}$ & $\begin{array}{c}2.96 \\
(2.97)\end{array}$ & $\begin{array}{c}4.88 \\
(4.88)\end{array}$ & $\begin{array}{c}4.85 \\
(4.85)\end{array}$ & \\
\hline
\end{tabular}

Note: the bond-valence parameters used are from Brese and O'Keeffe (1991). The sum values for $\mathrm{Zn}$ and $\mathrm{Cu}$ were calculated without taking into account the minor presence of vacancies on these sites (see text and Table 2)

an increase in the number of vacancies occurs along the solid solution series planerite $\left(\square \mathrm{Al}_{6}\left(\mathrm{PO}_{4}\right)_{2}\left(\mathrm{PO}_{3} \mathrm{OH}\right)_{2}(\mathrm{OH})_{8} \cdot 4 \mathrm{H}_{2} \mathrm{O}\right)$ - turquoise $\left(\mathrm{CuAl}_{6}\left(\mathrm{PO}_{4}\right)_{4}(\mathrm{OH})_{8} \cdot 4 \mathrm{H}_{2} \mathrm{O}\right)$ causes an increase of the protonation of the $\mathrm{PO}_{4}$ tetrahedra of the structure. Our results suggest that the necessary charge balance might also be achieved by introducing either additional $\mathrm{Cu}^{2+}$ or other cations in the partially occupied site at $(1 / 2,0,1 / 2)$.

The $\mathrm{AlO}_{6}$ octahedra and $\mathrm{PO}_{4}$ tetrahedra in both faustite and turquoise are somewhat distorted. The relatively large range of $\mathrm{Al}-\mathrm{O}$ and $\mathrm{P}-\mathrm{O}$ bond lengths reported for turquoise by Cid-Dresdner (1965) $(<\mathrm{P}-\mathrm{O}>1.54 \AA$, range $1.52-1.56 \AA$; $<\mathrm{Al}-\mathrm{O}>1.92 \AA$, range $1.81-2.16 \AA$ ) was only partially confirmed; the ranges found in the present study are slightly smaller (Table 4 ), and the $\mathrm{PO}_{4}$ tetrahedra are clearly more regular $(<\mathrm{P}-\mathrm{O}>1.532 \AA$, range $1.521-1.541 \AA)$. Very similar ranges are present in faustite $(\angle \mathrm{P}-\mathrm{O}\rangle$ $1.532 \AA$, range $1.516-1.541 \AA ;<\mathrm{Al}-\mathrm{O}>$ $1.904 \AA$, range $1.814-2.121 \AA$ ). The $\mathrm{PO}_{4}$ tetrahedra in chalcosiderite are comparable $(<\mathrm{P}-\mathrm{O}\rangle$ $1.536 \AA$ A , range 1.526-1.547 Аं; Giuseppetti et al., 1989).
The average $\mathrm{Al}(3)-\mathrm{O}$ bond distance in faustite, $1.913 \AA$, is slightly larger than those of $\mathrm{Al}(1)-\mathrm{O}$ and $\mathrm{Al}(2)-\mathrm{O}(1.896$ and $1.904 \AA$, respectively), but the distances are still identical within standard uncertainties. The $\mathrm{Al}(3) \mathrm{O}_{6}$ octahedron is clearly more regular than the $\mathrm{Al}(1) \mathrm{O}_{6}$ and $\mathrm{Al}(2) \mathrm{O}_{6}$ octahedra, and its shortest Al(3)-O bond, $1.887(4) \AA$, is considerably longer than those in the other two octahedra, $1.817(4)$ and $1.814(4) \AA$. Although this suggested the presence of very minor Fe on the Al(3) site, an occupancy factor refinement and the calculated bond-valence sums (Table 6) provided no convincing evidence for this. Nonetheless, in the final Fourier difference maps, residual electron density was only found close to $\mathrm{Al}(3)$ but not $\mathrm{Al}(1)$ or $\mathrm{Al}(2)$. The $\mathrm{Al}(3) \mathrm{O}_{6}$ octahedron in turquoise has a similar geometry (Table 4), although no possible substituent for $\mathrm{Al}(3)$ has been detected by the chemical analyses. A different $\mathrm{Fe}^{3+}$ - $\mathrm{Al}$ substitution model has been suggested for ferrian turquoise ('rashleighite'; Cid-Dresdner and Villarroel, 1972), where $\mathrm{Fe}^{3+}$ seemed to statistically occupy all three Al sites. On the other hand, during a 


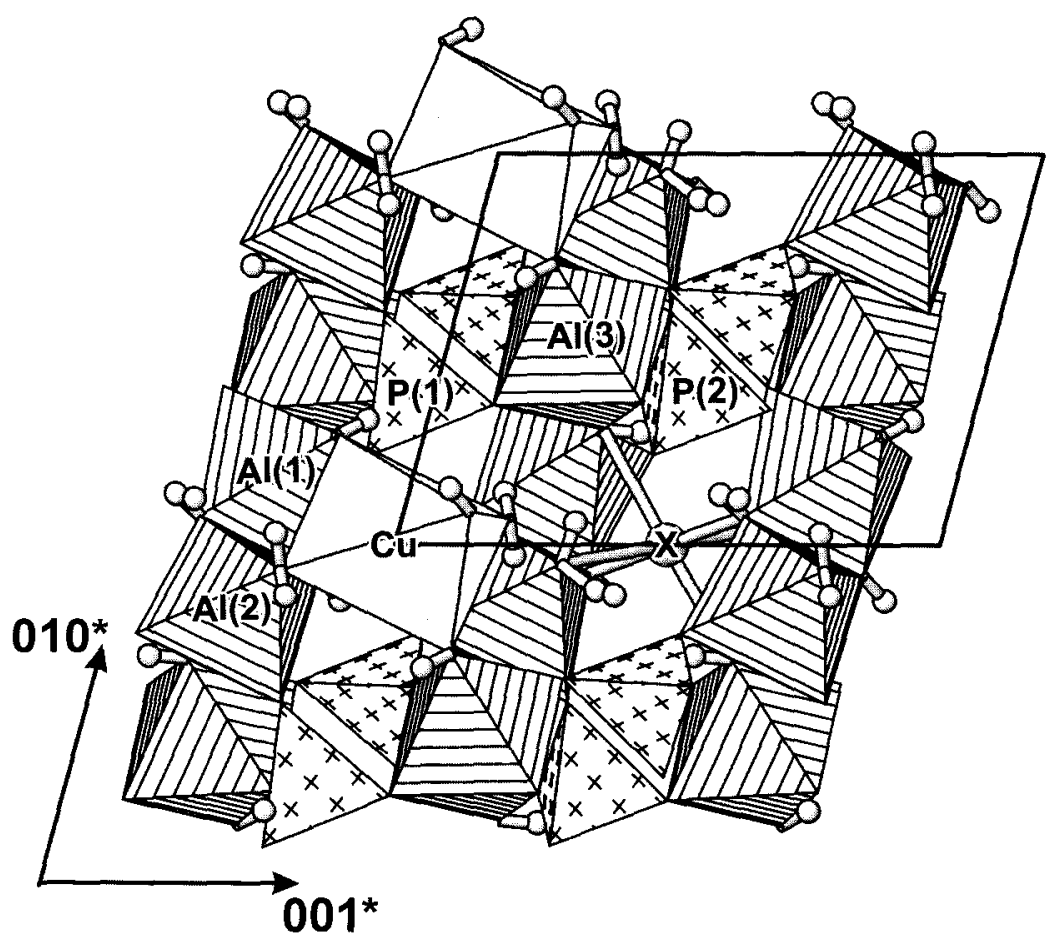

FIG. 2. View of the turquoise structure along the $a$-axis (the unit cell is outlined). $\mathrm{PO}_{4}$ tetrahedra are marked with crosses, $\mathrm{AlO}_{6}$ octahedra with parallel lines, and the distorted $\mathrm{CuO}_{6}$ polyhedron is shaded. The large grey sphere represents the partially occupied site (' $\mathrm{X}$ ') at the position $(1 / 2,0,1 / 2)$ (see text). The small grey spheres are $\mathrm{H}$ atoms.

structure analysis of an Al-bearing chalcosiderite, $\mathrm{Cu}\left(\mathrm{Fe}_{5.46}^{3+} \mathrm{Al}_{0.54}\right)\left(\mathrm{PO}_{4}\right)_{4}(\mathrm{OH})_{8} \cdot 4 \mathrm{H}_{2} \mathrm{O}$, Giuseppetti et al. (1989) found evidence that $\mathrm{Fe}$ prefers the most regular $(\mathrm{Fe}, \mathrm{Al}) \mathrm{O}_{6}$ octahedron, i.e. that octahedron which corresponds to $\mathrm{Al}(3) \mathrm{O}_{6}$ in faustite [note that $\mathrm{Fe}(1), \mathrm{Fe}(2 A)$ and $\mathrm{Fe}(2 B)$ of Giuseppetti et al. (1989) correspond to $\mathrm{Al}(3), \mathrm{Al}(1)$ and $\mathrm{Al}(2)$ in the present work].

\section{Hydrogen bonding}

Probable hydrogen bonds, listed in Table 5, show reasonable bond distances and angles which are similar for both faustite and turquoise. The hydrogen bonds are medium strong to very weak, with $\mathrm{O} . \mathrm{O}$ distances between 2.70 and $3.03 \AA$. The observed $\mathrm{O}-\mathrm{H} \cdots \mathrm{O}$ angles accord with the geometry of hydrogen bonds with variable strengths, although the unconstrained refinement of the $\mathrm{H}$ atom positions leads to increased uncertainties for these angles. Some of the bonds may be bifurcated (see Table 5) and the hydrogen bonding system appears to be flexible to some extent. The calculated bond-valence sums for the $\mathrm{O}, \mathrm{Oh}$ and $\mathrm{Ow}$ atoms indicate a 'mixed' ligand character for some of them (Table 6). The isotropic displacement parameters of the $\mathrm{H}$ atoms do, however, not suggest any pronounced disorder of the $\mathrm{H}$ positions. The partially occupied site in turquoise at $(1 / 2,0,1 / 2)$ may be the acceptor of $H$ bonds donated by the $H(4)$ and $H(8)$ atoms, if one assumes that the site is occupied by water molecules.

\section{Acknowledgements}

The authors thank Mr R. Bayerl of Stuttgart, Germany, for kindly lending the turquoise specimen to us. The faustite specimen was found by Mr Glyn Francis of Iron Knob, South Australia, who donated it to the South Australian Museum, Adelaide, South Australia. The careful and valuable comments of the referees A.G. Christy and L. Norén helped to improve the manuscript. 


\section{CRYSTAL STRUCTURE OF FAUSTITE}

\section{References}

Brese, N.E. and O`Keeffe, M. (1991) Bond-valence parameters for solids. Acta Crystallogr., B47, $192-7$.

Brown, I.D. (1996) VALENCE: a program for calculating bond valences. J. Appl. Crystallogr., 29, 479-80.

Brown, I.D. and Altermatt, D. (1985) Bond-valence parameters obtained from a systematic analysis of the inorganic crystal structure database. Acta Crystallogr., B41, 244-7.

Cid-Dresdner, H. (1965) Determination and refinement of the crystal structure of turquois, $\mathrm{CuAl}_{6}\left(\mathrm{PO}_{4}\right)_{4}(\mathrm{OH})_{8} \cdot 4 \mathrm{H}_{2} \mathrm{O}$. Zeits. Kristallogr., 121, 87-113.

Cid-Dresdner, H. and Villarroel, H.S. (1972) Crystallographic study of rashleighite, a member of the turquoise group. Amer. Mineral., 57, 1681-91.

Eby, R.K. and Hawthorne, F.C. (1993) Structural relationships in copper oxysalt minerals. I Structural hierarchy. Acta Crystallogr., B49, 28-56.

Erd, R.C., Foster, M.D. and Proctor, P.D. (1953) Faustite, a new mineral, the zinc analogue of turquois. Amer. Mineral., 38, 964-72.

Foord, E.E. and Taggart, J.E., Jr. (1998) A reexamina- tion of the turquoise group: the mineral aheylite, planerite (redefined), turquoise and coeruleolactite. Mineral. Mag., 62, 93-111.

Giuseppetti, G., Mazzi, F. and Tadini, C. (1989) The crystal structure of chalcosiderite, $\mathrm{CuFe}^{3+}{ }_{6}\left(\mathrm{PO}_{4}\right)_{4}(\mathrm{OH})_{8} \cdot 4 \mathrm{H}_{2} \mathrm{O}$. Neues Jahrb. Mineral. Mh., 227-39.

Pring, A., Kolitsch, U. and Francis, G. (2000) Additions to the mineralogy of the Iron Monarch deposit, Middleback Ranges, South Australia. Austral. $J$. Mineral., 6, 9-23.

Shape Software (1999) ATOMS for Windows and Macintosh V5.0. Kingsport, TN 37663, USA.

Sharma, K.B.N., Moorthy, L.R., Reddy, B.J. and Vedanand, S. (1988) EPR and electronic absorption spectra of a copper bearing turquoise mineral. Phys. Lett. A, 132, 293-7.

Sheldrick, G.M. (1997a) SHELXS-97, a program for the solution of crystal structures. University of Göttingen, Germany.

Sheldrick, G.M. (1997b) SHELXL-97, a program for crystal structure refinement. University of Göttingen, Germany.

[Manuscript received 21 February 2000:

revised 20 May 2000] 